Stabilized Spheromak Fusion Reactors

T.K. Fowler

April 4, 2007 
This document was prepared as an account of work sponsored by an agency of the United States Government. Neither the United States Government nor the University of California nor any of their employees, makes any warranty, express or implied, or assumes any legal liability or responsibility for the accuracy, completeness, or usefulness of any information, apparatus, product, or process disclosed, or represents that its use would not infringe privately owned rights. Reference herein to any specific commercial product, process, or service by trade name, trademark, manufacturer, or otherwise, does not necessarily constitute or imply its endorsement, recommendation, or favoring by the United States Government or the University of California. The views and opinions of authors expressed herein do not necessarily state or reflect those of the United States Government or the University of California, and shall not be used for advertising or product endorsement purposes.

This work was performed under the auspices of the U.S. Department of Energy by University of California, Lawrence Livermore National Laboratory under Contract W-7405-Eng-48. 


\title{
Stabilized Spheromak Fusion Reactors
}

T. K. Fowler

April 16, 2007

\begin{abstract}
The U.S. fusion energy program is focused on research with the potential for studying plasmas at thermonuclear temperatures, currently epitomized by the tokamakbased International Thermonuclear Experimental Reactor (ITER) but also continuing exploratory work on other plasma confinement concepts. Among the latter is the spheromak pursued on the SSPX facility at LLNL. Experiments in SSPX using electrostatic current drive by coaxial guns have now demonstrated stable spheromaks with good heat confinement, if the plasma is maintained near a Taylor state, but the anticipated high current amplification by gun injection has not yet been achieved. In future experiments and reactors, creating and maintaining a stable spheromak configuration at high magnetic field strength may require auxiliary current drive using neutral beams or RF power. Here we show that neutral beam current drive soon to be explored on SSPX could yield a compact spheromak reactor with current drive efficiency comparable to that of steady state tokamaks. Thus, while more will be learned about electrostatic current drive in coming months, results already achieved in SSPX could point to a productive parallel development path pursuing auxiliary current drive, consistent with plans to install neutral beams on SSPX in the near future. Among possible outcomes, spheromak research could also yield pulsed fusion reactors at lower capital cost than any fusion concept yet proposed.
\end{abstract}

\section{Introduction}

Experiments utilizing coaxial gun injection in the SSPX facility at LLNL have produced small spheromaks with toroidal-like confinement at temperatures up to $500 \mathrm{eV}$ [1]. Good confinement occurs inside a boundary called the separatrix that separates nominally closed magnetic flux surfaces from an open line flux core connected to the gun. For the stable configuration achieved thus far, the magnetic field inside the separatrix resembles the relaxed state predicted by Taylor [2]. As clarified by NIMROD 
simulations [3], after formation of the spheromak, stability is accomplished by reducing the gun current in the flux core to be sufficient to provide a stabilizing toroidal field inside the separatrix, but at a current below that required to inject magnetic helicity from the flux core across the separatrix. Thus the plasma inside the separatrix slowly decays. On the other hand, steady auxiliary current drive properly distributed across the profile could first build and then maintain the stable Taylor state, or any other stable state, indefinitely. Thus auxiliary current drive could be used to create and sustain stable spheromaks in steady state reactors, or during the burn phase of a pulsed reactor.

Three main issues determine the feasibility of spheromak reactors stabilized by auxiliary current drive: (1) the efficiency of current drive, (2) the extent to which current drive is required to stabilize the edge region near the separatrix, and (3) the current required for ignition. Current drive efficiency versus power balance is discussed in Sections 2 and 3, and edge stabilization in Section 4. Ignition conditions and spheromak scaling are discussed in Section 5. Access requirements to install auxiliary current drive are discussed briefly in Section 6, along with comments on potential advantages of spheromak reactor designs. Section 7 discusses computer modeling using the NIMROD code, including buildup to high magnetic fields, needed also to design a Proof-ofPrinciple experiment. Conclusions are presented in Section 8.

\section{Current Drive Efficiency}

Cordey [4] has published a formula for the efficiency of neutral beam current drive giving the driven current $\mathrm{I}$ in ratio to beam power $\mathrm{P}$ that fits experimental data such as that obtained on the DIIID tokamak at General Atomics. A comparison of neutral beam current drive and current drive by Electron Cyclotron Heating on DIIID gave comparable efficiencies, whereby $5 \mathrm{MW}$ of neutral beams produced $0.2 \mathrm{MA}$ of current, while $1-2 \mathrm{MW}$ of ECH produced 0.05MA.

The results of Cordey's calculation can be understood from the following simple relationship:

$$
\mathrm{I} / \mathrm{P}=\left(\mathrm{I}_{\mathrm{B}} \mathrm{N} / \mathrm{I}_{\mathrm{B}} \mathrm{E}_{\mathrm{B}}\right)\left(1-1 / \mathrm{Z}_{\mathrm{EFF}}\right)=\left(\mathrm{N} / \mathrm{E}_{\mathrm{B}}\right)\left(1-1 / \mathrm{Z}_{\mathrm{EFF}}\right)
$$


Here $I_{B}$ and $E_{B}$ are the beam current and energy, and $1 / Z_{E F F}$ represents partial cancellation of the ion current by electrons that are born with the beam speed but collide with background ions in the plasma with effective charge $Z_{E F F}$. The quantity $N=\left(v_{B} \tau / 2 \pi R_{M}\right)$ with beam speed $\mathrm{V}_{B}$ is the number of times beam ions circulate around the torus before slowing down by collisions with electrons, with beam speed $v_{B} \propto \sqrt{E}_{B}$, major radius $R_{M}$ and slowing down time $\tau \propto \mathrm{T}^{3 / 2} / \mathrm{n}$ with electron temperature $\mathrm{T}$ and density $\mathrm{n}$. Putting in numbers gives [4]:

$$
\mathrm{I} / \mathrm{P}=0.5\left(\mathrm{~T} / \mathrm{E}_{\mathrm{B}}\right)^{1 / 2}\left(\mathrm{~T} / \mathrm{n}_{20} \mathrm{R}_{\mathrm{M}}\right)\left(1-1 / \mathrm{Z}_{\mathrm{EFF}}\right) \quad \mathrm{MA} / \mathrm{MW}
$$

with $T$ and $E_{B}$ in $\mathrm{KeV}, \mathrm{R}_{\mathrm{M}}$ in meters and $\mathrm{n}$ in units of $10^{20} \mathrm{~m}^{-3}$. Based on Fokker-Planck calculations of beam ion collisions with the plasma, Cordey found an optimum injection energy for deuterium beams, $\mathrm{E}_{\mathrm{B}}=80 \mathrm{~T}$, giving $0.5\left(\mathrm{~T} / \mathrm{E}_{\mathrm{B}}\right)^{1 / 2}=0.06$. It is also necessary that $\mathrm{E}_{\mathrm{B}}$ be large enough for beam ions to penetrate the plasma.

For given reactor parameters $n, T$ and $R_{M}$, the current drive efficiency is determined by $\left(\mathrm{T} / \mathrm{E}_{\mathrm{B}}\right)^{1 / 2}$. Optimizing $\mathrm{E}_{\mathrm{B}}$ to penetrate into the plasma while also minimizing the power required to produce the beams has led to the development of $\approx$ $\mathrm{MeV}$ negative-ion-based beams for ITER, while current drive experiments on DIIID used $80 \mathrm{KeV}$ positive-ion-based neutral beams, and TFTR used $120 \mathrm{KeV}$ positive-ion-based beams. Beam penetration depends on $\mathrm{nR}_{\mathrm{M}}$ where $\mathrm{R}_{\mathrm{M}}=\mathrm{Aa}$ with aspect ratio $\mathrm{A}$ and minor radius a, while at a given temperature fusion power $\propto n^{2} R_{M} a^{2}$. Thus, for a given power:

$$
\mathrm{nR}_{\mathrm{M}} \quad \propto \quad \sqrt{(\mathrm{A} / \mathrm{a})}
$$

Thus it may be possible to design a compact spheromak reactor with $\mathrm{A}=1$ with somewhat lower beam energy than is required for ITER with $\mathrm{A} \approx 3$.

These comments about compact systems apply also to the spherical tokamak concept that is the basis for the NSTX experiment in Princeton, for which neutral beam current drive experiments are in progress. The main advantage of spheromaks is the absence of the central column required in a spherical tokamak needed to house TF coils that produce the toroidal field and, in NSTX, the solenoid producing inductive current 
drive, though electrostatic current drive by a coaxial gun is also being explored on NSTX. As in all tokamaks, it is the toroidal field in NSTX that accounts for its inherent stability.

The disadvantage of spheromaks is the possibility of residual MHD instability that disturbs flux surfaces, even for the "stabilized" case, and additional current drive power that would be needed if stabilization is required at the edge in order to maintain a Taylor state everywhere. On the other hand, if a spheromak can be made as stable as already appears to be the case in SPPX, any reactor designer would opt to omit the central column, in order to simplify the divertor and above all to simplify blanket and neutron shielding design.

It may or may not be necessary to stabilize the edge, as discussed in Section 4, and in any case the additional current drive power required to stabilize the edge is only a factor 2 or so greater than that for the tokamak-like stable case. To see this, we average $\mathrm{N}$ $\propto \mathrm{T}^{3 / 2}$ over the temperature profile giving:

$$
\mathrm{N}=\quad \mathrm{N}_{\mathrm{S}} \int_{0}^{\mathrm{a}}\left(2 \mathrm{rdr} / \mathrm{a}^{2}\right)|\mathrm{j}(\mathrm{x}) / \mathrm{j}(0)|\left(1-\mathrm{x}^{2}\right)^{3 / 2}
$$

Here $\mathrm{T} \propto\left(1-\mathrm{x}^{2}\right)$ with $\mathrm{x}=\mathrm{r} / \mathrm{a}$ giving $\left.\mathrm{N}=\mathrm{N}_{\mathrm{S}}\left(1-\mathrm{x}^{2}\right)\right]^{3 / 2}$; and we have weighted the integral by the current density $\mathrm{j}$ being driven by the beam. For a current density peaked on axis, as in a tokamak or the potentially stable spheromak discussed in Section $4, \mathrm{~N}=\mathrm{N}_{\mathrm{S}}$ giving Eq. (2) above. For the parabolic temperature profile and constant j, the average $<\mathrm{N}\rangle \geq$ $2 / 5 \mathrm{~N}_{\mathrm{S}}$ even if $\mathrm{j}$ is roughly uniform as in a Taylor state.

\section{Power Balance in Stabilized Spheromak Reactors}

We shall first discuss a spheromak maintained in a stable Taylor state with flat $\lambda$ profile, as in present SSPX experiments, where $\lambda=\mu_{0} \mathrm{j} / \mathrm{B}$ is proportional to the current density $\mathrm{j}$. The possibility that stability may allow a $\lambda$ profile that is "sagging" toward the separatrix is discussed in Section 4 and Appendix A.

To illustrate, we consider the steady state spheromak reactor design of Hagenson and Krakowski [5], reviewed in Ref. [6]. Using the numbers in Refs. [5] and [6], we find for the Hagenson-Krakowski design: 


\begin{tabular}{|c|c|c|c|}
\hline $\mathrm{R}$ & $=$ & $3 \mathrm{~m}$ & (spheromak diameter) \\
\hline B & $=$ & $5 \mathrm{~T}$ & (nominal average magnetic field) \\
\hline I & $=$ & $47 \mathrm{MA}$ & (toroidal current) \\
\hline $\mathrm{n}_{20}$ & $=$ & 2.26 & (averaged density) \\
\hline$\tau_{\mathrm{E}}$ & $=$ & $0.4 \mathrm{~s}$ & (energy confinement time) \\
\hline $\mathrm{E}_{\mathrm{MAG}}$ & $=$ & $1435 \mathrm{MJ}$ & (magnetic energy) \\
\hline $\mathrm{P}_{\text {FUSION }}$ & $=$ & $2775 \mathrm{MW}$ & (alpha plus neutron) \\
\hline $\mathrm{P}_{\text {ELECtRIC }}$ & $=$ & $1100 \mathrm{MW}$ & (at $40 \%$ net including blanket) \\
\hline $\mathrm{P}_{\text {Oнміс }}$ & $=$ & $19 \mathrm{MW}$ & (inside separatrix) \\
\hline
\end{tabular}


The power required to drive the current by neutral beams is given by Eq. (2) for the "stable" case discussed in Section 4, or this multiplied by $5 / 2$ to maintain a "stabilized" Taylor state, as assumed in the Hagenson-Krakowski design. For a nominal beam energy $E_{B}=1000 \mathrm{KeV}, R_{M}=0.5 R$ and $Z_{E F F}=2$, we obtain for the stable case $I / P=$ $0.25 \mathrm{MA} / \mathrm{MW}$ giving:

$$
\begin{aligned}
\mathrm{P}_{\text {BEAM }} & =[(47 \mathrm{MA}) / 0.25] & = & & \text { Stable } \\
& \approx 5 / 2 \times 188 & = & 470 \mathrm{MW} & \text { Stabilized }
\end{aligned}
$$

While net power would be produced, this range of $\mathrm{N}$ corresponds to a large recirculation of electric power, mainly because of the inefficiency of non-inductive current drive, which is true also for tokamaks. Setting aside beam production efficiency, $\mathrm{P}_{\text {BeAM }} / \mathrm{P}_{\text {ELECTRIC }}=42 \%$ to maintain a Taylor state (the Stabilized case) and $17 \%$ for the most optimistic Stable case. However, this is within a factor of 2 or so of expected performance in ITER and steady state tokamak reactors, and at a toroidal current $\mathrm{I}=47$ MA that is more than double what is believed necessary to maintain ignition in tokamaks. The effect of the actual ignition condition on spheromak reactor scaling is discussed in Section 5 .

\section{Edge Stabilization and the Divertor}

The power balance is most favorable if, instead of the Taylor state with a flat $\lambda$ profile, the plasma remained stable even if the $\lambda$ profile "sags" to zero near the separatrix -- approaching the "Stable," tokamak-like case of Section 3.

The existence of a stable case with sagging $\lambda$ profile has been explored briefly with some favorable evidence (see Appendix A), but much more work is required. Besides improving the power balance, the existence of a stable state allows the same freedom to design a divertor for the spheromak reactor as for the tokamak, but simpler due to easy access from the ends of the device (as in mirror machines). By divertor, here we refer not only to the flux core but specifically to the return of the bias flux $\Phi$ as it flows along the wall of the quasi-spherical vacuum chamber. One concern is the 
thickness $\Delta$ of this return flux, referred to as the "scrapeoff" layer. For a spheromak with a cylindrical vacuum chamber of radius $R$ and length $L=R$ :

$$
\Delta \quad=\quad(\Phi / \pi \mathrm{R})
$$

For the stable case not requiring any current in the flux core or divertor, we are free to maintain constant $\Delta$ during buildup by programming $\Phi$, much as the PF coils are programmed in tokamaks. First efforts to study the effects of programming $\Phi$ using the NIMROD code, but for a different purpose, are discussed in Ref. [7].

For the stabilized case in which it is assumed that a Taylor state must be maintained even into the flux core and divertor, additional power is required to maintain current on open field lines, using the electrostatic gun. As an illustration, let us assume a nominal 30\% efficiency for electrostatic injection (by optimizing the power supply compared to SSPX) and, in order not to impact the power balance too much, a power of $50 \mathrm{MW}$ to maintain the required current, thus allowing $15 \mathrm{MW}$ ohmic loss which is comparable to $\mathrm{P}_{\text {OHмIC }}$ inside the separatrix. Equating these losses gives [with $\eta_{\mathrm{E}}=\mathrm{AT}_{\mathrm{E}}{ }^{-3 / 2}$ on open lines and an average value $\langle\eta\rangle=\mathrm{A}(\mathrm{T} \sqrt{\mathrm{E}})^{-1}$ inside the separatrix]:

$$
\mathrm{j}_{\mathrm{E}}^{2} \Delta \pi \mathrm{R}^{2} \eta_{\mathrm{E}}=\mathrm{j}_{\mathrm{E}}^{2} \Delta \pi \mathrm{R}^{2} \mathrm{AT}_{\mathrm{E}}^{-3 / 2} \approx \pi^{2} \mathrm{R}^{3}<\eta \mathrm{j}^{2}>=\pi^{2} \mathrm{R}^{3} \mathrm{~A}\left(\mathrm{~T} \sqrt{ } \mathrm{T}_{\mathrm{E}}\right)^{-1}<\mathrm{j}^{2}>
$$

where $\mathrm{j} \propto \lambda$ is the current density and the geometric factors are the approximate divertor and interior volumes. Then:

$$
\Delta / \mathrm{R} \quad \approx \quad 3 \pi\left(\mathrm{T}_{\mathrm{E}} / \mathrm{T}\right) \quad \approx 0.1
$$

with nominal $<\mathrm{j}^{2}>/ \mathrm{j}_{\mathrm{E}}{ }^{2}=3$ and $\left(\mathrm{T} / \mathrm{T}_{\mathrm{E}}\right)=100$ (perhaps too high when heating on open lines due to heat transport out of the hot plasma is taken into account).

Note that, while we found the power balance to be more or less acceptable even for the stabilized case, this case does require peaking the beam power near the separatrix - either by separate beams or by spreading beam angles appropriately. This also implies a large number of beam ion orbits skimming the separatrix, probably satisfactory at steady 
state. For the Hagenson-Krakowski steady state design, the minor radius a is about 20 times the gyroradius $r_{\mathrm{L}}$ for a $1 \mathrm{MeV}$ deuterium ion at the weakest field near the outer wall (giving $\mathrm{a} / \mathrm{r}_{\mathrm{L}} \geq 20$ everywhere); and "banana" drift excursions are comparable to gyroradii in spheromaks. We will return to this issue in discussing buildup in Section 7.

\section{Ignition Conditions and Stabilized Spheromak Reactor Scaling}

Energy confinement in SSPX appears to be encouraging. The beta achieved in SSPX is within a factor of 2 of that for classical heat transport with perfect flux surfaces and $\mathrm{T}_{\mathrm{e}}=\mathrm{T}_{\mathrm{i}}$.

The most relevant information for extrapolating to future experiments and reactors concerns confinement in the hot "core" near the magnetic axis, in order to avoid including edge losses that are better ascribed to maintaining the edge magnetic field as discussed in Ref. [8] and Section 4. A detailed analysis is given in Ref. [1]. Here we note that, to obtain a local power balance giving the observed $\beta \approx 10 \%$ in the core, we require:

$$
\tau_{\mathrm{E}}=3 / 2 \beta \tau_{\Omega}=0.008 \mathrm{~s}
$$

This result is obtained from $\eta \mathrm{j}^{2}=3 / 2 \mathrm{nT} / \tau_{\mathrm{E}}$ using $\mu_{\mathrm{o}} \mathrm{j}=\lambda \mathrm{B}$ with $\lambda=5 / \mathrm{R}=10$, and also $\beta$ $=\left(2 \mu_{\mathrm{o}} \mathrm{nT} / \mathrm{B}^{2}\right) \approx 0.1$ and $\tau_{\Omega}=\left(\mu_{\mathrm{o}} / 2 \eta \lambda^{2}\right)$ with $\eta=1.13 \times 10^{-7}$ at $\mathrm{T}=500 \mathrm{eV}$.

To extrapolate, we first apply tokamak L-mode scaling using the formula in Ref. [9], given by:

$$
\tau_{\mathrm{E}} \quad=\quad 0.023\left(\mathrm{~A}^{0.2}\right) \mathrm{I}_{\mathrm{MA}}{ }^{0.96} \mathrm{R}_{\mathrm{M}}{ }^{1.83}\left(10 \mathrm{n}_{20}\right)^{0.4} \mathrm{P}_{\mathrm{MW}}{ }^{-0.73}
$$

where factors not applicable to spheromaks have been omitted; and A is the ion mass in ratio to hydrogen and $R_{M}=0.5 R=a$, the minor radius. To apply Eq. (9) to SSPX, we take for the power the ohmic heating in the core giving $\mathrm{P}=\mathrm{I}^{2} \mathrm{R}_{\Omega}=0.15 \mathrm{MW}$ with $\mathrm{R}_{\Omega}=$ $\left(2 \pi \mathrm{a} / / \pi \mathrm{a}^{2}\right)=10^{-6} \mathrm{ohm}$ at $500 \mathrm{eV}$. Using this and SSPX parameters I $=0.4 \mathrm{MA}$ inside the separatrix and $\mathrm{R}_{\mathrm{M}}=0.25, \mathrm{~A}=1$ and $\mathrm{n}_{20} \approx 1$, we obtain $\tau_{\mathrm{E}}=0.007 \mathrm{~s}$, similar to Eq. (8). Extrapolating to the parameters of Section 3 for the Hagenson-Krakowski reactor design, for the minimum current drive power $\mathrm{P}=188 \mathrm{MW}$ we obtain $\tau_{\mathrm{E}}=0.2 \mathrm{~s}$, which is half that 
required for power balance ignoring heating by the beams. For ITER-98P(y,2) scaling, we obtain $\tau_{\mathrm{E}}=1 \mathrm{~s}$.

That L-mode scaling is only marginally favorable for reactors is not surprising, based on similar studies for tokamaks. The actual energy confinement may be better or worse, depending on the extent of degradation of confinement due to residual MHD turbulence that destroys flux surfaces, thus requiring higher I to reach ignition. The greatest leverage in reducing I is by stabilizing resistive MHD modes to the fullest extent possible. Resistive MHD instability can now be studied on NIMROD, as discussed in Section 7.

\section{Access for Auxiliary Current Drive, Reactor Design}

Before discussing NIMROD simulations, we pause to make a few comments about access for current drive in reactor designs, and potential advantages of spheromak reactors. We note that, whereas coaxial guns can be mounted at the geometric axis where divertor flux must escape in any case, neutral beam injection requires line-of-sight access through the vacuum chamber wall, and RF requires space to mount antennas inside the machine. For steady state reactors, some thought has already been given to mounting neutral beams on compact devices, in conjunction with the Field Reversed Mirror concept that motivated the earlier Beta II neutral beam injection experiment at LLNL [10].

Pulsed spheromak reactors not requiring external magnets may offer a unique path to lower development cost and lower capital cost $[11,12]$. Access for a coaxial gun in pulsed spheromak reactors was discussed in Refs. [11] and [12], taking into account liquid lithium or FLIBE "blankets" that permit the high power density operation leading to lower reactor cost. Successful auxiliary current drive in a pulsed reactor would probably require aiming neutral beams through the same points of access -- a hole at each end near the axis. The concept in Ref. [12] allowing the liquid blanket to close the hole during fusion burn would not apply if injection is required to stabilize the burn. Thus pulsed reactors with auxiliary current drive pose formidable design issues, but with a high payoff for success. High power density pulsed spheromak reactors could yield reactor capital costs as low as $\$ 15 \mathrm{M}$ for the fusion “nuclear island”[12]. 


\section{Simulating Current Buildup and Stability Using NIMROD}

An important part of the SSPX success story is the validation of the NIMROD code to study the effects of resistive MHD modes, now to the point that NIMROD should be utilized as the best available tool to model future experiments, as in Ref. [7]. Thus far, the greatest discrepancy concerns electron temperatures in the simulations -- about a factor 2 below those in SSPX - perhaps due to sensitivity to details for the classical electron heat transport along field lines included in NIMROD.

The simplest way to initiate studies of current drive on NIMROD would be adding a specified force or electric field $\mathbf{E}_{\mathrm{B}}(\mathbf{x})$ with sign chosen to drive current in Ohm's Law. Based on experience in stabilizing MST [7A], fine tuning may not matter so that a simple spatial distribution for $\mathbf{E}_{\mathrm{B}}(\mathbf{x})$ may suffice to show a positive trend, even if adjustment is needed to obtain the quietest state and best heat confinement. Ideally, besides its toroidal component, $\mathbf{E}_{\mathrm{B}}(\mathbf{x})$ should contain a poloidal component, which requires aiming beams at various angles. In the simulation, this could be accomplished by choosing $\mathbf{E}_{\mathrm{B}}(\mathbf{x})$ to be parallel to the mean field $\mathbf{B}_{\mathbf{0}}$.

We can see the physical meaning of $\mathbf{E}_{\mathrm{B}}(\mathbf{x})$ by examining Ohm's Law for neutral beam injection, given by:

$$
-\partial \mathbf{A} / \partial \mathrm{t} \quad=\quad \eta\left\{\mathbf{j}-\mathbf{j}_{\mathbf{i}}\left(1-1 / \mathrm{Z}_{\mathrm{EFF}}\right)\right\}
$$

and multiplying by $\lambda^{2} / \mu_{\mathrm{o}}$ gives the current buildup equation:

$$
\partial \mathbf{j} / \partial \mathrm{t} \quad=\quad \tau^{-1}\left\{\mathbf{j}_{\mathbf{i}}\left(1-1 / \mathrm{Z}_{\mathrm{EFF}}\right)-\mathbf{j}\right\}
$$

where $\tau=\left(\mu_{0} / \lambda^{2} \eta\right)$ is the decay time for the current. To actually calculate buildup, we must solve for $\mathbf{j}_{\mathrm{i}}$, which is the current density due to injected beam ions, satisfying:

$$
\left.\partial \mathbf{j}_{\mathrm{i}} / \partial \mathrm{t} \quad=\quad \tau_{\mathbf{B}}^{-1} \mathbf{j}_{\mathbf{B}}(\mathbf{x})\right)-\tau_{\mathbf{S}}^{-1} \mathbf{j}_{\mathrm{i}}
$$

where $\mathbf{j}_{\mathbf{B}}(\mathbf{x})$ is the current density of injected beam ions $\left(I_{\mathbf{B}}=\int \mathrm{d} \mathbf{S} \cdot \mathbf{j}_{\mathbf{B}}(\mathbf{x})\right)$. Also $\tau_{\mathbf{S}}$ is the 
slowing down time and $\tau_{\mathbf{B}}=\left(2 \pi R_{M} / v_{B}\right)$. An induction term omitted in Eq. (12) has a small effect on the Ohm's Law but a beam source must be added to the heat equation (see Appendix B).

Since $\tau_{\mathbf{B}}<<\tau_{\mathbf{S}}<<\tau$, we solve Eq. (12) in steady state giving $\mathbf{j}_{\mathrm{i}}=\mathrm{Nj}_{\mathbf{B}}(\mathbf{x})$ with $\mathrm{N}=$ $\left(\tau_{\mathbf{S}} / \tau_{\mathbf{B}}\right)$ as defined in Section 2. Substituting this into Eq. (11) gives for the buildup equation:

$$
\partial \mathbf{j} / \partial \mathrm{t} \quad=\quad \tau^{-1}\left\{\mathrm{Nj}_{\mathbf{B}}(\mathbf{x})\left(1-1 / \mathrm{Z}_{\mathrm{EFF}}\right)-\mathbf{j}\right\}
$$

Substituting the steady state $\mathbf{j}_{\mathbf{i}}=\mathbf{N j}_{\mathbf{B}}(\mathbf{x})$ into Eq. (10) and treating the last term as an electric field gives:

$$
\begin{array}{rll}
\mathbf{E}_{\mathrm{B}}(\mathbf{x}) \quad & \quad \eta \mathbf{j}_{\mathbf{B}}(\mathbf{x})\left(1-1 / \mathrm{Z}_{\mathrm{EFF}}\right) \\
& \propto & {\left[\left(\sqrt{\mathrm{E}_{\mathrm{B}}} / \mathrm{nR}\right)\left(\mathrm{Z}_{\mathrm{EFF}^{-}} 1\right)\right] \mathbf{j}_{\mathbf{B}}(\mathbf{x})}
\end{array}
$$

where in the second line we use $\eta \propto \mathrm{Z}_{\mathrm{EFF}} \mathrm{T}^{-3 / 2}$ and $\mathrm{N} \propto \mathrm{T}^{3 / 2}$ as in Section 2. Substituting Eq. (14) into the Ohm's Law of NIMROD yields a current buildup equation equivalent to Eq. (13).

In steady state, Eq. (13) gives $\mathbf{j} \propto \mathrm{Nj}_{\mathbf{B}}(\mathbf{x}) \propto \mathrm{T}^{3 / 2} \mathbf{j}_{\mathbf{B}}(\mathbf{x})$. Hence any distribution of $\mathbf{j}$ needed to achieve a stable state can be obtained by properly shaping the injected current profile $\mathbf{j}_{\mathrm{B}}(\mathbf{x})$ as noted in the Introduction. Achieving a Taylor state with roughly constant $\mathbf{j}$ requires $\mathbf{j}_{\mathrm{B}}(\mathbf{x}) \propto \mathrm{T}^{-3 / 2}$. Once the beams take over, if the system is stable $\mathrm{T}$ will grow due to beam heating and current buildup will continue until heat transport causes $\mathrm{T}$ to saturate.

The condition that neutral beam current drive takes over from the gun is $\mathrm{Nj}_{\mathbf{B}}(\mathbf{x})\left(1-1 / \mathrm{Z}_{\mathrm{EFF}}\right)>\mathbf{j}$ at the temperature and current density of the target. Based on performance already achieved in SSPX, suitable target parameters might be $\beta=5-10 \%$, $\mathrm{T}=200 \mathrm{eV}$ and $\mathrm{n}_{20}=2.26$ as in the reactor design of Section 3 , or $\mathrm{T}=500 \mathrm{eV}$ and $\mathrm{n}_{20}=1$ as in SSPX now. Starting from a few $100 \mathrm{eV}$ and building to $20 \mathrm{KeV}$, the current and flux amplification could be 15-to-50 fold, similar to cases explored in Ref. [7]. 
A different issue limiting current amplification is the minimum field $\mathrm{B}$ required to confine beam ions. For $1000 \mathrm{KeV}$ beams, in Section 4 we found $\mathrm{a} / \mathrm{r}_{\mathrm{L}}=20$ near the outer wall at full field. If the same beams are used to build up the current (as would be convenient), the maximum current amplification $\approx 10$ in order to maintain $\mathrm{a} / \mathrm{r}_{\mathrm{L}}>1$ at the beginning of neutral beam injection. Then gun injection must produce I $\approx 5$ MA (about 5 times that in SSPX), which is feasible in a device of reactor size with an efficiently designed power supply delivering $\mathrm{E}_{\mathrm{MAG}}=15 \mathrm{MJ}$ into the flux conserver.

Finally, for pulsed reactors the crucial issue is the efficiency of recreating the magnetic field after each pulse [11]. The buildup efficiency can be increased by decreasing the density during buildup followed by fuel injection to initiate the burn phase.

\section{Conclusions}

Experiments on SSPX have already demonstrated at least one way to achieve a stable spheromak at interesting temperatures, though electrostatic current drive giving these results has not yet achieved high flux or current amplification. Plans to install neutral beams on SSPX are consistent with a different path to achieve high currents, using neutral beam auxiliary current drive (demonstrated in the DIIID tokamak) that could in principle both build up the magnetic field level and maintain stability. Another possibility is RF current drive that also receives attention elsewhere in the fusion program.

The high temperatures achieved in SSPX, together with the rich physics of magnetic relaxation being learned in SSPX, is drawing strong support outside LLNL, including a recent endorsement that SSPX is the only experiment in its class now ready to propose advanced funding to the Proof of Principle (POP) level [14].

Besides achieving high temperatures, the other major success in the SSPX program is validation of the NIMROD code in modeling phenomena associated with resistive MHD instability, now to the point that NIMROD should be used in planning future experiments, as it has so successfully been used in interpreting experimental results. 
A two-pronged approach employing NIMROD and experiments now planned for SSPX should provide the basis for a POP proposal, and eventually pilot the way toward the very promising kind of fusion reactors discussed in this paper.

\section{Acknowledgement}

I would like to thank Bick Hooper for numerous helpful discussions in the course of this work.

References:

[1] (a) H. S. McLean et al, Phys. Review Letters 88, 125004 (2002). (b) H. S. McLean et al. Phys. of Plasmas 13, 056105 (2006). The achievement of T = $500 \mathrm{eV}$ was reported at the meeting, cited in Ref. [14].

[2] J. B. Taylor, Rev. Modern Physics 58, 741 (1986).

[3] B. I. Cohen et al. Phys. of Plasmas 12, 0556106 (2005).

[4] J. G. Cordey, Plas, Phys. and Controlled Fus. 26, 123 (1984),

[5] R. L. Hagenson and R. A. Krakowski, Fus. Tech. 8, 16011 (1985).

[6] T. K. Fowler, “A Spheromak Ignition Experiment Reusing Mirror Fusion Test Facility (MFTF) Equipment,” UCID-ID-114696, Sept. 28, 1993.

[7] E. B. Hooper et al, "Achieving high flux amplification in a gun-driven, fluxcore spheromak," UCRL-JRNL-228353, 2007.

[7A] C. R. Sovinec and S.C. Prager, Nucl. Fus. 39, 777 (1999).

[8] T. K. Fowler, J. S. Hardwick and T. R. Jarboe, Comments Plas. Phys. Controlled Fusion, 16, 91 (1994).

[9] S. M. Kaye et al., Nucl. Fusion 37, 1303 (1997).

[10] R. F. Post, "Expermental Basis of Mirror-Confinement Physics,"Fusion, E. Teller, Editor, Academic Press, New York, 1981, Vol. 1, Part A, Chapter 6.

[11] T. K. Fowler, D. D. Hua, E. B. Hooper, R. W. Moir and L. D. Pearlstein, "Pulsed Spheromak Fusion Reactors," Comments Plas. Phys. Contr. Fusion, Comm. Mod. Phys. 1 C, 83 (1999). 
[12] T. K. Fowler and P. F. Peterson, "Conceptual Design of a Pulsed Spheromak Fusion Reactor," Univ. of Calif. Dept, of Nuclear Eng. Report, UC-BFE-052, Nov. 5, 1998.

[13] T. K. Fowler and R. Gato, "Magnetic relaxation and hyper-resistivity during helicity injection," in process of submission for publication.

[14] OFES Budget Meeting, Washington D.C. Spring, 2007.

\section{Appendix A}

Stable Spheromaks: Pulsed and Steady State Reactors

T. K. Fowler

January 26, 2006

Work since my seminar with Pearlstein January 20 confirms the general idea that stable states of the spheromak must exist, even with a "sagging" $\lambda$ profile falling to zero at the edge -- hence one not requiring gun current to sustain it.

As noted at the seminar, up to now interest in spheromaks has been motivated by the stability of the exact Taylor state and the simplicity of creating spheromaks in the Taylor state by gun injection [1]. From the point of view of fusion reactors, this physics interest in spheromaks was reinforced by Krakowski's observation that steady state spheromak reactors sustained by gun injection and helicity transport can achieve high fusion power gain despite the power required to sustain a Taylor state where the temperature drops near the edge [2]. Pulsed reactors maintaining a Taylor state by helicity transport from the magnetic axis out to the edge during decay were also shown to be attractive [3]. High power gain is NOT consistent with maintaining a gun current on open field lines (e.g. to stabilize the spheromak, as in current SSPX high temperature shots), if the required current exceeds $<1 \%$ of the toroidal current of the spheromak.

New evidence has raised questions whether helicity transport, which intermittently opens field lines, can be consistent with maintaining good energy confinement. Here we note that stable states exist that do not rely on helicity transport, and we make a preliminary assessment of their usefulness to fusion as motivation for further work. 
In the absence of helicity transport, these stable states arise naturally as poloidal current dies to near-zero at the edge when gun sustainment of the edge ceases, as in the decaying state of a pulsed reactor, or a steady state sustained by non-inductive current drive. A similar situation arises for RFP's with inductive drive, again in principle giving rise to a stable state with a "sagging" $\lambda$ profile with $\lambda \approx 0$ at the edge.

That stable states in RFP's exist with sagging lambda profiles was demonstrated in straight-cylinder approximation by Robinson [4]. Recently Pearlstein has obtained similar results for spheromaks, for tearing in cylinder approximation and for ideal modes in actual SSPX geometry using Corsica for equilibria and DCON to analyze stability [5]. Earlier Jardin found spheromaks stable to ideal MHD modes, even with a significant gap between the plasma and the wall, thus allowing room for a divertor to remove impurities [6]. Pearlstein finds that DCON predicts stability for all ideal modes in SSPX for both peaked and flattened $\lambda$ profiles, but the maximum allowed gap has not yet been calculated.

Pearlstein finds that stability to tearing occurs only for $\lambda$ profiles that are relatively flat in the interior, with a maximum value $\lambda_{\text {MAX }}$ not too much above the lowest eigenvalue $\lambda_{\mathrm{o}}$ (Taylor state) over most of the volume. In Pearlstein's calculations using equilibria from Corsica fitted to a cylinder for the tearing calculation, for a profile $\lambda=$ $\lambda_{\text {MAX }}\left(1-\psi^{\mathrm{N}}\right)$ with poloidal flux $\psi$ a marginally stable state occurs around $\mathrm{N}=5$ giving $\lambda_{\mathrm{MAX}} / \lambda_{\mathrm{o}} \approx 1.2$, whereas a peaked profile with $\mathrm{N}=1$ or 2 gives a few tearing modes and $\lambda_{\mathrm{MAX}} / \lambda_{\mathrm{o}} \approx 2$.

That Pearlstein's tearing results are likely to stand up in an exact treatment in toroidal geometry is suggested by a comparison with eigenvalues for kink-like curleigenstates in SSPX, from which actual perturbation solutions can be constructed. Eigenvalues are like energy levels, instability being a transition from a symmetric equilibrium state to a twisted field of lower energy. For SSPX with flux conserver lengthto-radius $L / R=1$, the lowest such eigenvalue $\lambda_{1}$ gives $\lambda_{1} / \lambda_{\mathrm{o}} \approx 1.1$ (compared to $\lambda_{\mathrm{MAX}} / \lambda_{\mathrm{o}} \approx$ 1.2 above) and the largest possible ratio $\lambda_{1} / \lambda_{0}$ is almost the same, occurring at $\mathrm{L} / \mathrm{R}=0.8$ [7].

We conclude that the aspect ratio of the cylindrical flux conserver in SSPX is nearly optimal, and any stable state -- other than an exact Taylor state -- is likely to have 
a sagging $\lambda$ profile, flat over most of the volume. A flattish $\lambda$ profile may be consistent with adequate confinement in spheromak reactors, if the edge region is stable as claimed above. The flat profile implies grad $\mathrm{p}=$ zero in the interior, with heat leak across an edge of thickness $\Delta<<$ a and fusion power production throughout the volume. Reactors with these characteristics have been considered in the past, the main consequence of the steep edge being a higher toroidal current to reach ignition, by a factor of order $(\mathrm{a} / \Delta)^{2 / 3}$ if gyroBohm transport is dominant, as is believed to the be case in tokamaks. While any increase in required toroidal current in ITER would be very costly, this is much less important for compact spheromaks, perhaps increasing the required current from about 30 MA in Refs. [2 and 3] to, say, 50 MA for Pearlstein's $\mathrm{N}=5$ profile. (A better estimate could be made by inserting Pearlstein's profile into the SPHERE heat transport code in straight-cylinder geometry.)

A greater concern is the tendency for resistive decay to distort the system away from the stable state. Left to its own devices, a flattish profile would decay toward a peaked profile, perhaps exciting global tearing modes that tend to open field lines everywhere in NIMROD simulations to date. This issue can only be explored using NIMROD, including heat transport.

Some form of control may be necessary to maintain the stable state. Control could be applied via non-inductive current drive, in a Steady State reactor, or in a Pulsed Reactor with just enough current drive to maintain stability during the burn. The same non-inductive drive could become the means for building up a large current in the spheromak, as in the earlier Beta II program.

Since the main concern is avoiding formation of a peaked profile, one possibility might be to apply non-inductive current drive toward the edge rather than at the magnetic axis. This would create a hollow current profile, probably exciting instability that would only fill in the hole up to the level of the flattish stable profile discussed above. Stifling the dynamo at the edge might require both poloidal and toroidal current drive, which could be accomplished by skewing the direction of the injection of neutral beams.

One might also consider a compromise with a solid conductor at the geometric axis, as in NSTX, but carrying much less current, hence more feasible mechanically. The current would be reversed, as in an RFP, with just enough current to partially reverse the 
field, if that aids stability in the interior. In principle this case is included in Ref. [4], and it would not be too difficult to analyze this case in Corsica. In this regard, we note that, in one sense, spheromaks are more stable than RFP's with large aspect ratio, and compact RFP's might be more stable than spheromaks. This hierarchy arises from the strong stabilizing effects of compact geometry, expressed through a stabilizing term in $\delta \mathrm{W}$ that is proportional to $\left[\mathrm{m}^{2}+\mathrm{n}^{2}\left(\mathrm{r}^{2} / \mathrm{R}^{2}\right)\right]$ with poloidal and toroidal mode numbers $\mathrm{m}$ and $\mathrm{n}$, and major radius $R$.

In a Pulsed Reactor, it would be desirable to retain to the extent possible the simplicity of building up current by gun injection rather than neutral beams [8]. For this case, and for SSPX experiments utilizing the gun, all ideas mentioned here require a transition from the "downhill-in- $\lambda$ " profile produced by the gun to a sagging $\lambda$ profile.

Exploring this transition could become an important new goal for SSPX. Evidence of instability during such a transition already appears in some experiments and NIMROD runs, with a corresponding dump of the heat due to the global nature of the modes. Experiments in SSPX together with NIMROD simulations are well-suited to study ways of minimizing losses during the transition, the best outcome being the creation of a state that remains stable after the gun current (and bias flux?) have been reduced to zero.

Finally, the stability analyses cited above, based on $\delta \mathrm{W}$ for zero pressure, should survive at finite pressure. Even high $\beta$ remains possible regarding ideal modes $[3,6]$. Finite pressure does introduce new resistive modes [6], which might, however, yield beneficial S -scaling for helicity transport as originally hoped [9] (but only if helicity is transported faster than heat leaks out).

\section{Summarizing:}

Stable states for spheromaks exist other than the Taylor state obtained by gun injection and helicity transport. Finding ways to access these states and determining their utility for fusion reactors requires more modeling on NIMROD. Successful scenarios on NIMROD could suggest new paths for SSPX. These new directions, together with a continuing effort to build up and sustain current by gun injection, would be complementary in exploring the full potential of spheromaks for fusion research. 


\section{References}

[1] J. B. Taylor, Rev. Mod. Phys. 58, 741 (1986).

[2] R. L. Hagenson and R. A. Krakowski, Fus. Tech. 8, 16011 (1985).

[3] T. K. Fowler, D. D. Hua, E. B. Hooper, R. W. Moir and L. D. Pearlstein, Comm. Plas. Phys.Contr. Fusion/Comm. Mod. Phys. 1, C, 83 (1999).

[4] D. C. Robinson, Nuc. Fusion 18, 939 (1978).

[5] L. D. Pearlstein, Seminar, Jan. 20, 2006, and private communications (memo in preparation).

[6] S. C. Jardin, Nucl. Fusion 22, 620 (1982).

[7] E. C. Morse, with thanks for data he provided using methods in his paper, Jour. Math. Phys. 46, 113511 (2005).

[8] T. K. Fowler, "Homopolar Gun for Pulsed Spheromak Fusion Reactors II," UCRL-TR-2204727, June, 2004.

[9] R.H. Cohen, H. L. Berk, B. I. Cohen, T. K. Fowler, E. B. Hooper, L. L. Lodestro, E. C. Morse, L. D. Pearlstein, T. D. Rognlien, D. D. Ryutov, C. R. Sovinec and S. Woodruff, Nucl. Fusion 43, 1220 (2003).

\section{Appendix B.}

Ohm's Law and the Heat Equation with the Ohkawa Current

The electric field $\mathbf{E}_{\mathbf{B}}(\mathbf{x})=\eta \mathbf{j}_{\mathbf{i}}\left(1-1 / Z_{\mathrm{EFF}}\right)$ in Eq.(14) means a term on the right side of Eq. (10), analogous to $\eta \mathbf{j}$, neoclassical corrections to the resistance, or a bootstrap current. As for the bootstrap current, which can build up the magnetic field, there is an implied coupling to other equations giving the source of energy for the change in field. Energy exchanges can be important for the Ohkawa current.

The Ohm's Law of Eq. (10) is the sum of electron and ion momentum equations weighted to give $\partial \mathbf{j} / \partial \mathrm{t}$, which is then dropped in comparison with the induction field $\partial \mathbf{A} / \partial t$. The issue concerns how to calculate $\mathbf{j}_{\mathrm{i}}$ due to injected ions appearing in Eq. (10), for application to a one-fluid code like the spheromak version of NIMROD. In the main text, this is done via Eq. (11) which is the parallel ion momentum equation only taking into account collisions with electrons. This omits the inductive $-\partial \mathbf{A} / \partial \mathrm{t}$, which controls the energy exchange with the field, as is already implied by Eq. (11). Retaining the induction field term gives in quasi-steady state: 


$$
\mathbf{j}_{\mathrm{i}} \quad=\quad \mathrm{N} \mathbf{j}_{\mathrm{B}}(\mathbf{x})+\left(\mathrm{n}_{\mathrm{B}} \mathrm{e}^{2} / \mathrm{m}_{\mathrm{B}}\right) \tau_{\mathrm{S}} \mathbf{E}_{||} \quad=\quad \mathrm{N} \mathbf{j}_{\mathrm{B}}(\mathbf{x})+\left(\mathrm{n}_{\mathrm{B}} / \mathrm{n} \eta\right) \mathbf{E}_{||}
$$

Here $\mathrm{n}_{\mathrm{B}}$ and $\mathrm{m}_{\mathrm{B}}$ are the beam density and ion mass obtained in constructing the beam ion equation contributing to Ohm's Law and again $\tau_{\mathrm{S}}=\left(\mathrm{m}_{\mathrm{B}} / \nu_{\mathrm{ei}} \mathrm{m}_{\mathrm{e}}\right)$ is the ion slowing down time.

Introducing Eq. (B1) into Eq. (10) gives Eq. (13) in the main text aside from a small correction to $\mathbf{E}_{\mid}$of order $\left(\mathrm{n}_{\mathrm{B}} / \mathrm{n}\right)\left(1-1 / \mathrm{Z}_{\mathrm{EFF}}\right)$. Conversely, if during buildup we neglect $\eta \mathbf{j}$ in Eq. (10) giving $\mathbf{E}_{||}=-\eta N \mathbf{j}_{\mathrm{B}}(\mathbf{x})\left(1-1 / \mathrm{Z}_{\mathrm{EFF}}\right)$, then substituting this $\mathbf{E}_{||}$into Eq. (B1) gives the steady state solution of Eq. (12) used to derive Eq. (13), aside from a correction of the same order. Thus Eq. (13) correctly describes buildup of current by neutral beam injection, the important induction effect appearing in $1 / \tau \propto \eta$.

The energy required for buildup of the field comes form $\mathbf{j} \cdot \mathbf{E}$, which becomes negative for large enough $\mathbf{j}_{\mathrm{i}}$ for field growth to occur, by the criterion of Section 7 . This same term negative $\mathbf{j} \cdot \mathbf{E}$ appearing in the heat equation then cools the temperature, increases $\eta$ and stops the growth. The correct formulation must add to $\mathbf{j} \cdot \mathbf{E}$ in the heat equation a beam term giving:

$$
\mathbf{j} \cdot \mathbf{E}+\mathrm{j}_{\mathrm{B}}(\mathbf{x})\left(\mathrm{E}_{\mathrm{B}} / \pi \mathrm{Re}\right)=\eta \mathrm{j}^{2}+\mathrm{j}_{\mathrm{B}}(\mathbf{x})\left\{\left(\mathrm{E}_{\mathrm{B}} / \pi \mathrm{Re}\right)-\eta \mathrm{Nj}\left(1-1 / \mathrm{Z}_{\mathrm{EFF}}\right)\right\}
$$

This introduces as a secondary condition for buildup $\left\{\left(\mathrm{E}_{\mathrm{B}} / \pi \mathrm{Re}\right)-\eta \mathrm{Nj}\left(1-1 / \mathrm{Z}_{\mathrm{EFF}}\right)\right\}>0$ so that $\mathrm{T}$ grows, whereby the steady state of Eq. (13) can give higher and higher $\mathbf{j}$ as $\mathrm{N} \propto$ $\mathrm{T}^{3 / 2}$ increases. Note that, while the condition of Section 7 depends on $\mathrm{T}$ (and through this determines a suitable target plasma for buildup), the secondary condition above does not depend on $\mathrm{T}$ directly since $\eta \mathrm{N}$ does not, but it does depend on $\mathrm{E}_{\mathrm{B}}$ (for which the Cordey optimum is $E_{B}=80 \mathrm{~T}$ in steady state). This secondary condition is satisfied for the buildup scenario for the Hagenson-Krakowski reactor design, so that the condition of Section 7 used to establish the reactor buildup scenario is in fact the dominant one. 